\title{
Bullatacin Triggered ABCB1-Overexpressing Cell Apoptosis via the Mitochondrial-Dependent Pathway
}

\author{
Yong-Ju Liang, Xu Zhang, Chun-Ling Dai, Jian-Ye Zhang, Yan-Yan Yan, Mu-Sheng Zeng, \\ Li-Ming Chen, and Li-Wu Fu
}

State Key Laboratory of Oncology in South China, Cancer Center, Sun Yat-Sen University, Guangzhou 510060, China

Correspondence should be addressed to Li-Wu Fu, fulw@mail.sysu.edu.cn

Received 20 February 2009; Accepted 11 May 2009

Recommended by Dominic Fan

This paper was to explore bullatacin-mediated multidrug-resistant cell apoptosis at extremely low concentration. To investigate its precise mechanisms, the pathway of cell apoptosis induced by bullatacin was examined. Bullatacin causes an upregulation of ROS and a downregulation of $\Delta \Psi_{m}$ in a concentration-dependent manner in ABCB1-overexpressing KBv200 cells. In addition, cleavers of caspase-9, caspase-3, and PARP were observed following the release of cytochrome c from mitochondria after bullatacin treatment. However, neither cleavage of caspase- 8 nor change of expression level of bcl-2, bax and Fas was observed by the same treatment. Pretreating KBv200 cells with N-acetylcysteine, an antioxidant modulator, resulted in a significant reduction of ROS generation and cell apoptosis induced by bullatacin. Bullatacin-induced apoptosis was antagonized by z-LEHD-fmk, a caspase- 9 inhibitor, but not by z-IETD-fmk, a caspase- 8 inhibitor. These implied that apoptosis of KBv200 cells induced by bullatacin was associated with the mitochondria-dependent pathway that was limited to activation of apical caspase- 9 .

Copyright (๑) 2009 Yong-Ju Liang et al. This is an open access article distributed under the Creative Commons Attribution License, which permits unrestricted use, distribution, and reproduction in any medium, provided the original work is properly cited.

\section{Introduction}

Multidrug resistance (MDR) is now recognized as one of the most common causes of failure in cancer chemotherapy. The MDR phenotype renders cross-resistance to structurally and functionally unrelated antitumor agents. This phenomenon often causes overexpression of MDR1 gene that encodes a $170-\mathrm{KD}$ transmembrane glycoprotein named as $\mathrm{P}$ glycoprotein (P-gp, ABCB1) [1].

Importantly, in addition to its role as an efflux pump, ABCB1 regulates programmed cell death mediated by chemotherapeutic agents, serum starvation, UV irradiation, as well as ligation of the cell surface death receptors Fas and tumor necrosis factor (TNF) receptor. Johnstone et al. [2] demonstrated that functional $\mathrm{ABCB} 1$ inhibited the activation of caspase- 8 and -3 following Fas ligation and this inhibitory effect could be reversed by $\mathrm{ABCB} 1$ antagonists, such as specific anti-ABCB1 monoclonal antibodies. The alterations in apoptotic pathways would confer MDR cell resistance to conventional chemotherapeutic agents such as doxorubicin and vincristine [3]. Therefore, ABCB1 may play a dual role in regulating cell death induced by these stimuli via (i) removing the toxins from the cell and (ii) inhibiting the activation of caspases- 8 and -3 but not caspase-9. So it has been postulated that MDR cells were sensitive to apoptosis induced by a mitochondria-dependent pathway.

Up to today, strategies aimed at reversing MDR have principally focused on inhibition or modulation of $A B C B 1$ activity. Many MDR modulators have been identified, some undergoing clinical trials, but currently none is in clinical use. Novel anticancer drugs with efficiency to MDR cells present another important strategy for overcoming MDR. Recently, extracts prepared from a variety of plants have been demonstrated to possess the ability in triggering the mitochondria-dependent apoptotic pathway [4].

Bullatacin, a compound with an adjacent bis-tetrahydrofuran ring structure of annonaceous acetogenins, isolated from the plant family annonaceae, is a promising novel lead compound of anticancer agents. Functionally, bullatacin exhibits potent bioactivities via inhibiting the complex I of mitochondria and the NADH oxidase of plasma membrane in tumor cells and depletion of ATP levels [5]. Furthermore, the ubiquinone-linked NADH oxidase, constitutively expressed in the cell membrane of cancer cells, but only 


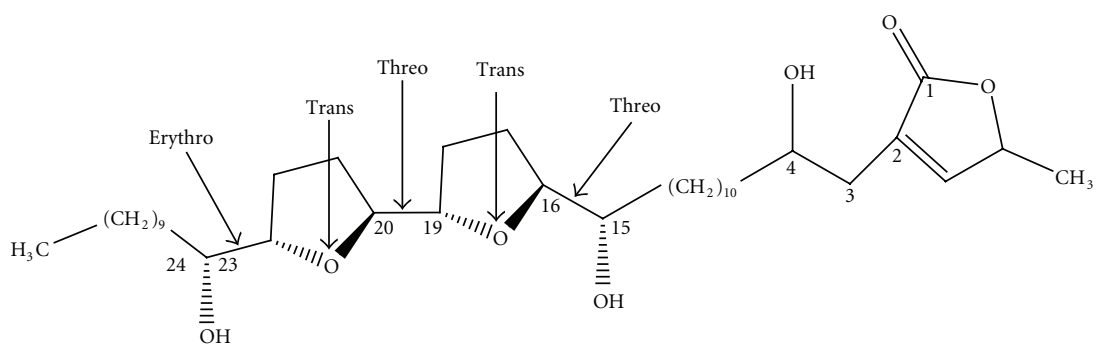

(a)

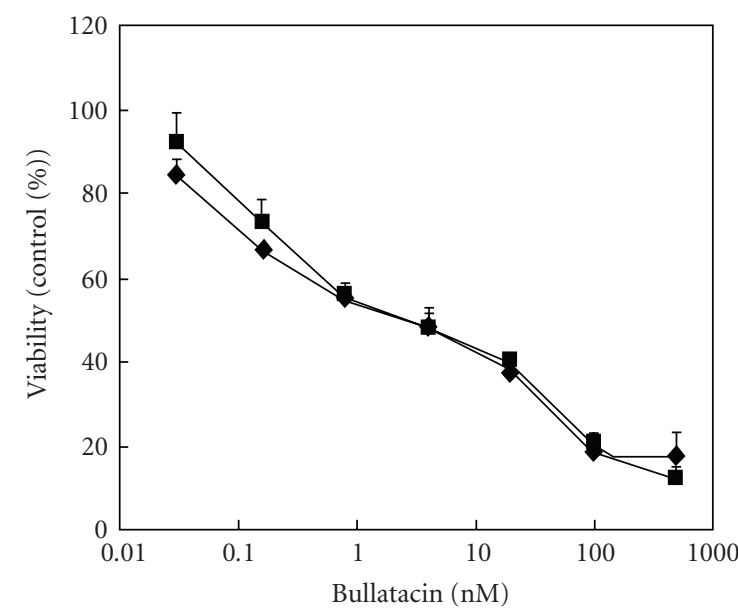

(c)

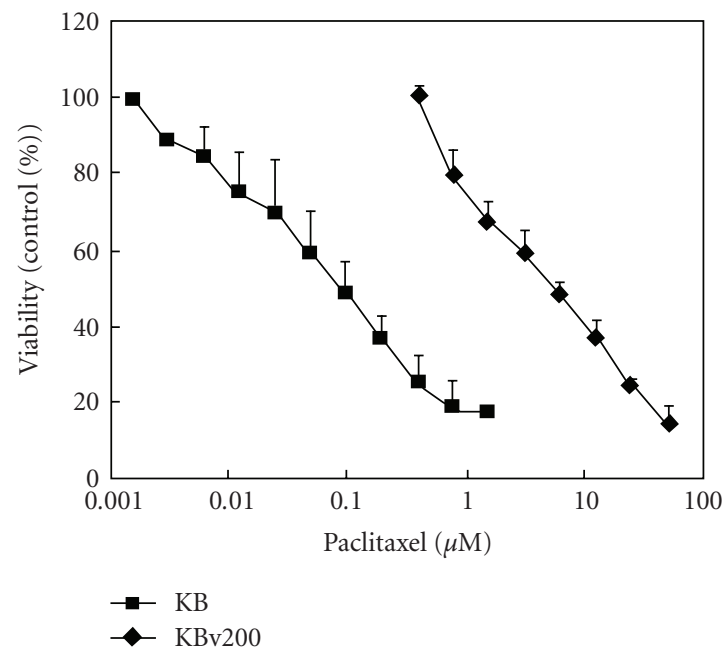

(e)

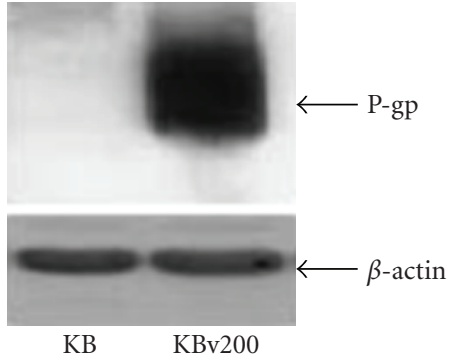

(b)

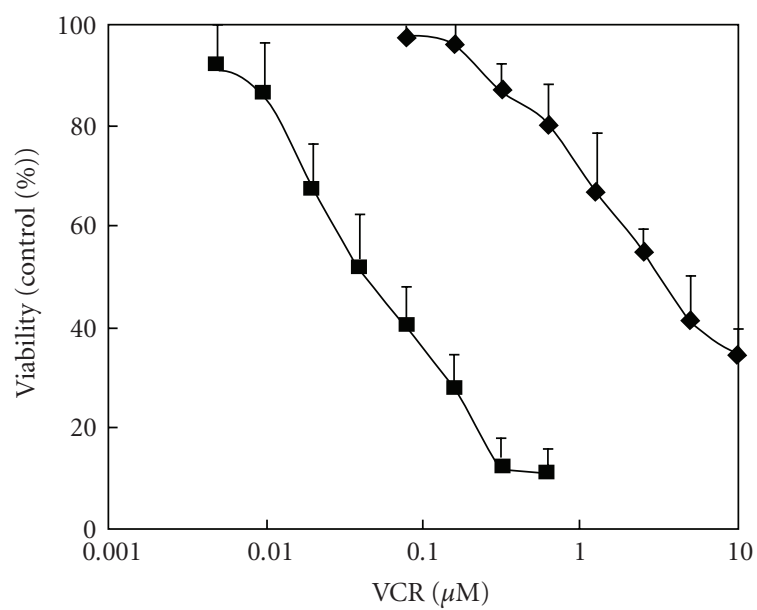

(d)

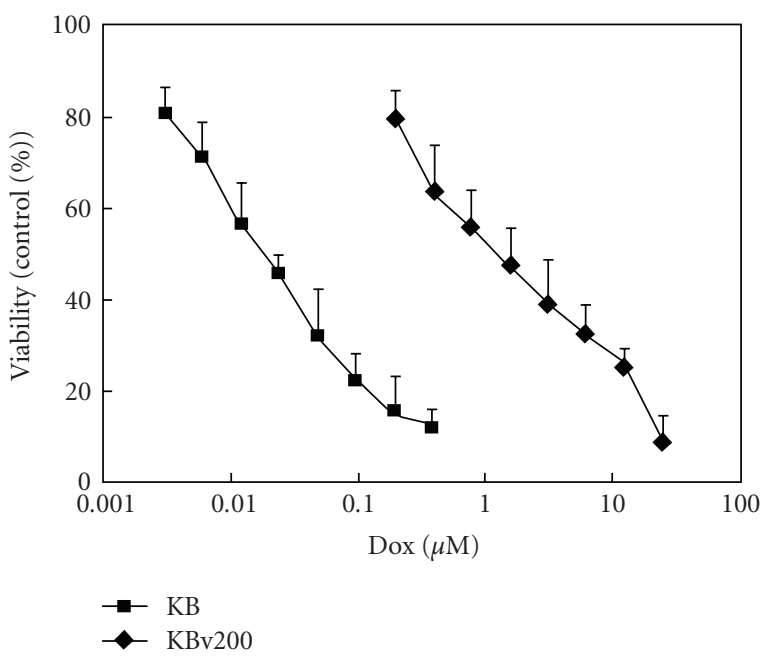

(f)

Figure 1: The structure of bullatacin (a), the overexpression of ABCB1 in KBv200 cells (b), equal amount of protein from various cells was loaded for Western blot as description in Section 2; the cytotoxicity of bullatacin (c), VCR (d), paclitaxel (e) and Dox (f) in KBv200 and KB cells. Cell survival was determined by MTT assay as described in Section 2. Data represent means and standard errors of at least a triplicate determination. 


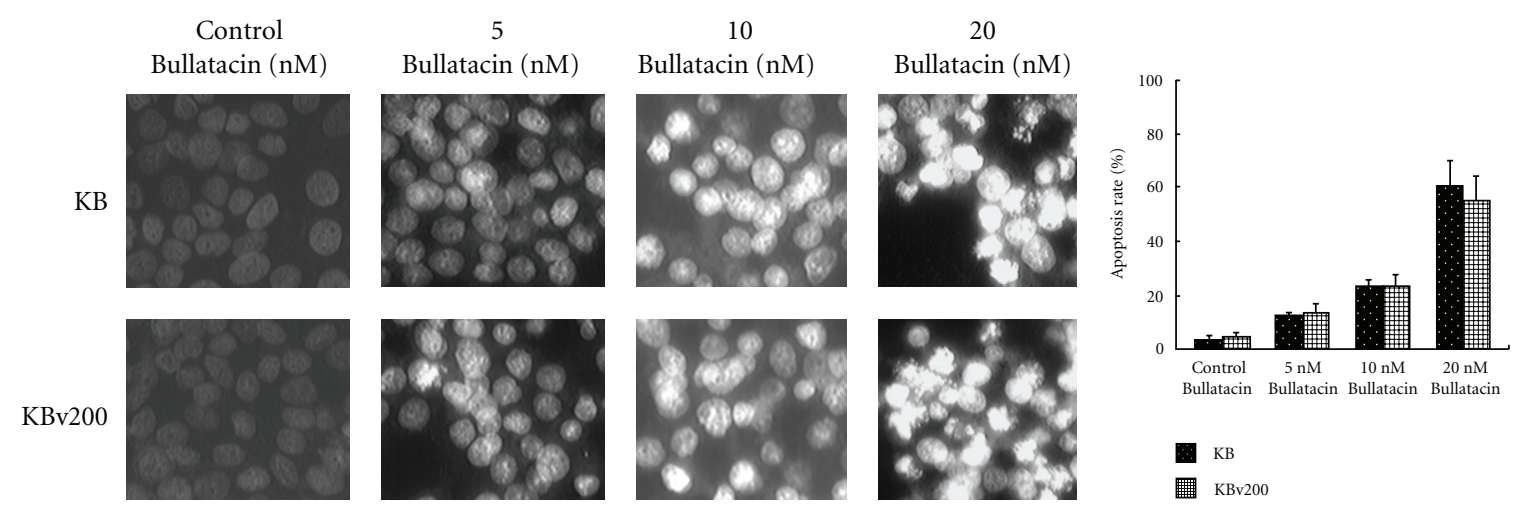

(a)

(b)
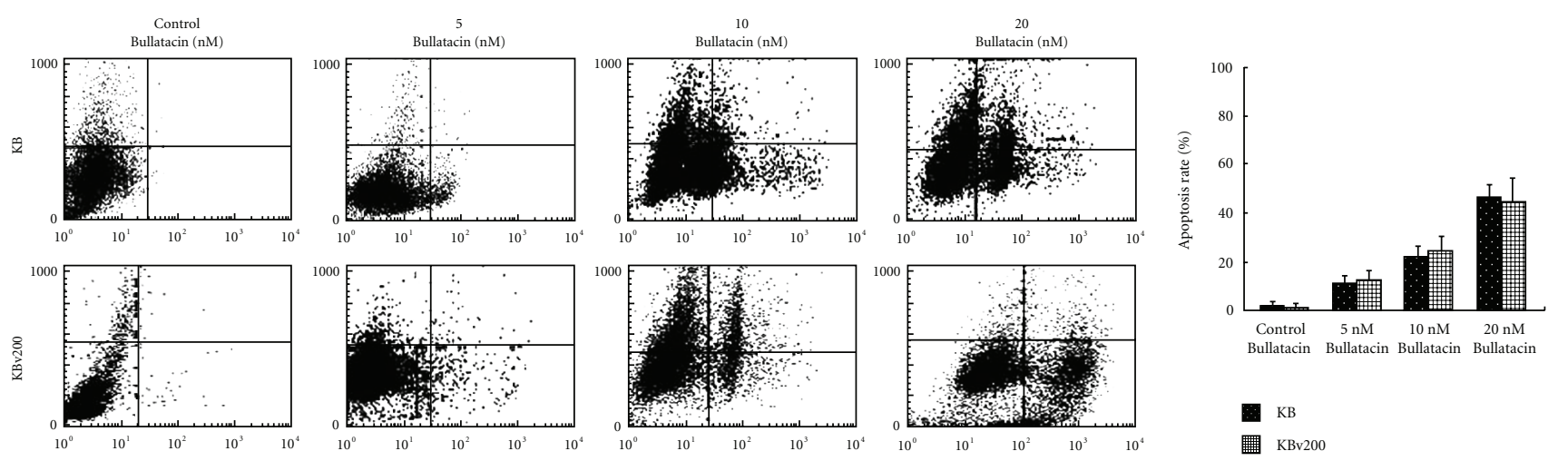

(c)

(d)
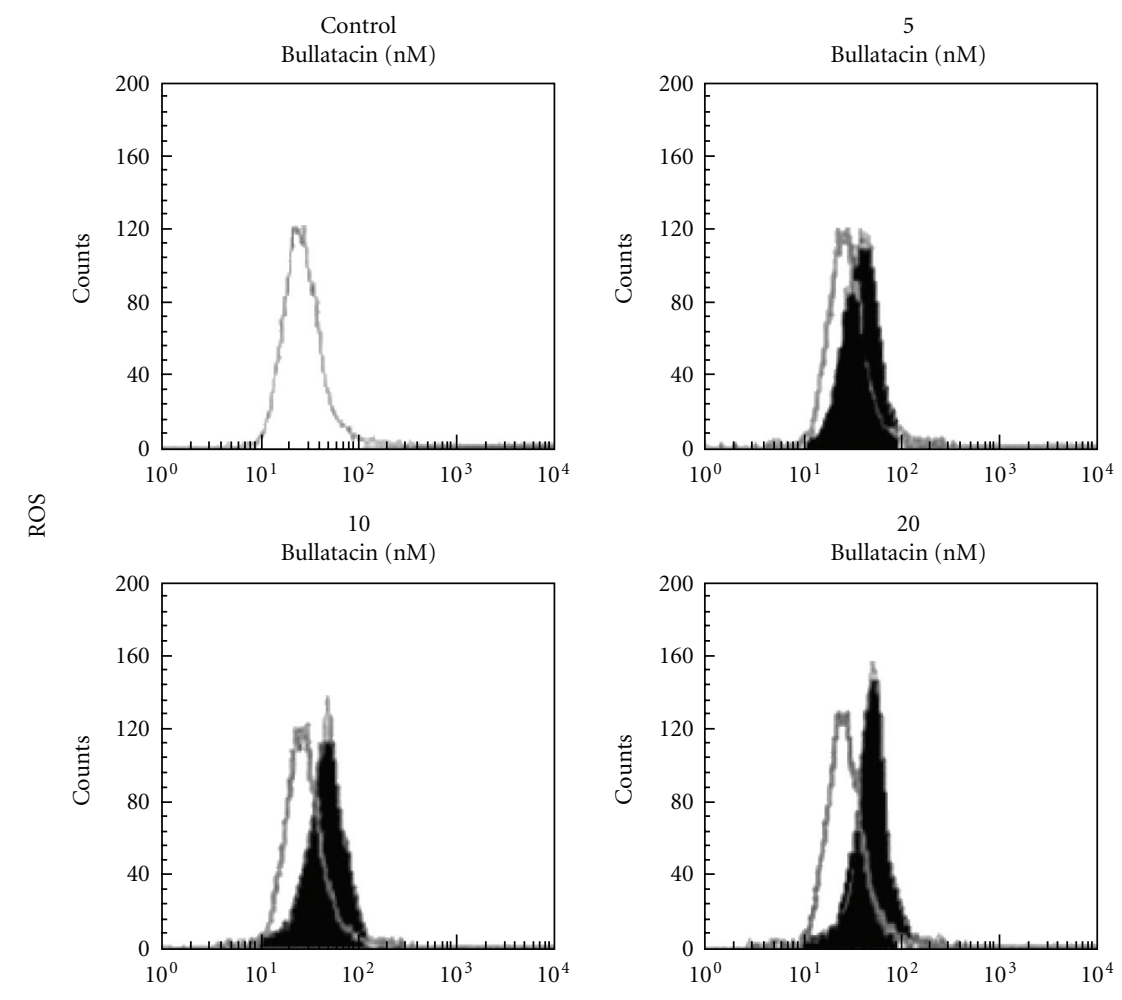

(e)

Figure 2: Continued. 

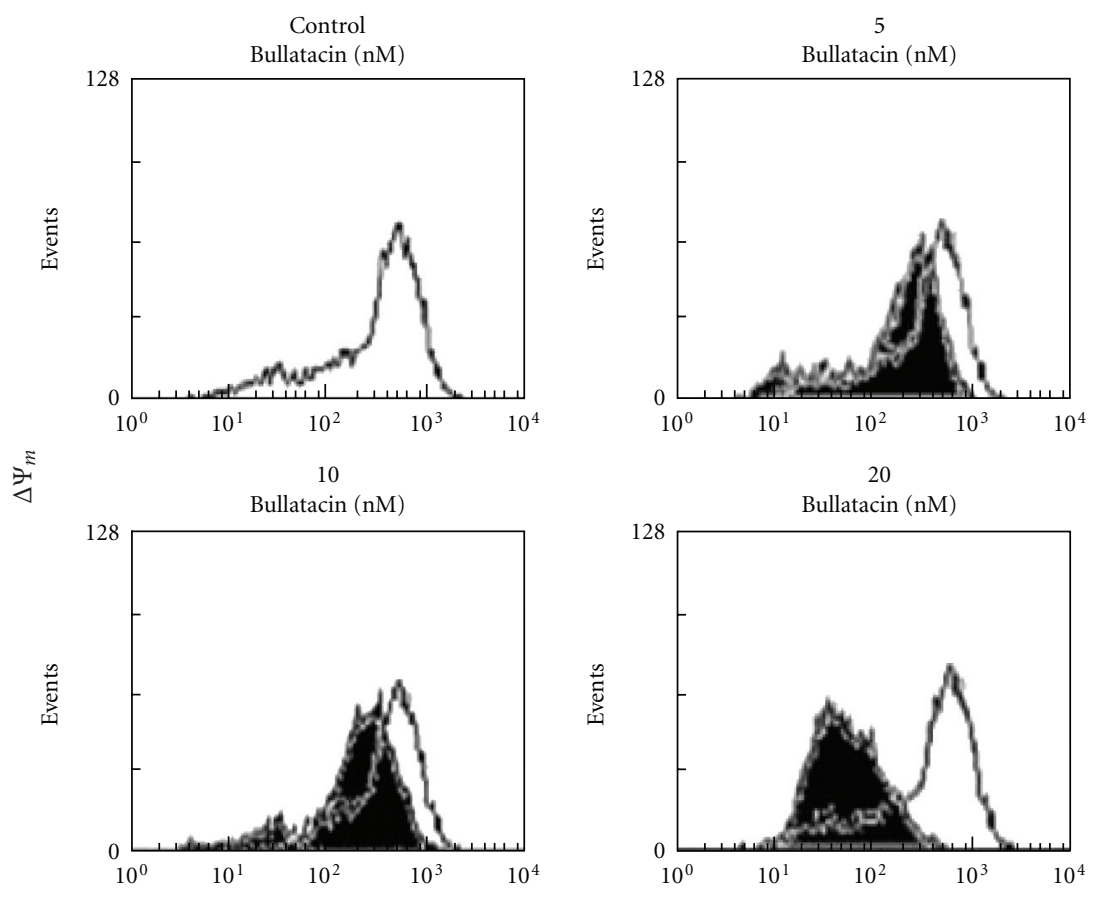

(f)

FIGURE 2: Apoptosis, ROS level and mitochondrial transmembrane potential $\left(\Delta \Psi_{m}\right)$ were determined in $\mathrm{KB}$ or KBv200 cells after treatment with bullatacin. (a) Apoptotic morphology of KB and KBv200 cells $(\times 400)$ was observed by Hoechst 33258 staining. The experiments were performed three times and a representative experiment is shown. (b) The apoptotic rates of KB and KBv200 cells were calculated with identified apoptosis cells by Hoechst 33258 stain dividing to accounted all cells (more than 2000 cells) after treated with bullatacin for 48 hours. Data represented means and standard errors of at least a triplicate determination. (c) Apoptotic cells were detected with Annexin V/PI after bullatacin treatment for 48 hours. The left-upper quadrant represents cells stained mainly by propidium iodide, while the right bottom quadrant represents cells stained mainly by Annexin V (apoptotic cells). The top right quadrant represents cells stained by both PI and Annexin V (secondary necrosis). The experiments were performed three times and a representative experiment is shown. (d) The apoptotic rates of KB and KBv200 cells was examined with Annexin V/PI after treated with bullatacin for 48 hours. Data represented means and standard errors of at least a triplicate determination. (e) ROS levels detection in KBv200 cells after treatment with bullatacin for 12 hours. Relative ROS levels were represented by the fold DCF intensity compared with untreated cells. The samples were duplicated and the data is a representative of three experiments. (f) Assessment of $\Delta \Psi_{m}$ collapse in KBv200 cells. The collapse of $\Delta \Psi_{m}$ was indicated by a reduction of $\mathrm{DiOC}_{6}$ intensity because the cells lost $\mathrm{DiOC}_{6}$ staining. Data represented means and standard errors of at least a triplicate determination.

transiently in that of normal cells, is also inhibited by bullatacin [6]. Importantly, bullatacin has shown potential high cytotoxicity in vitro and antitumor activity in vivo [69]. However, it is not yet clear how bullatacin inhibits the growth of $50 \%$ of grown MDR cancerous cells at extremely low concentrations in vitro. Could bullatacin induce MDR cell apoptosis? Which pathway of cell apoptosis induced by bullatacin will be involved in? Further research on the functioning of mitochondria will hopefully lead to a better assessment for the applicability of bullatacin.

\section{Materials and Methods}

2.1. Materials. Bullatacin was isolated from the seed of the Annona atemoya by Professor W.S. Chen (South China Institute of Botany, Chinese Academy of Sciences). Its structure is shown in Figure 1(a). MTT, Hoechst 33258, Annexin VFITC and PI were products of Sigma Chemical Co. from Genewindows Co. (Guangzhou, China). Mouse monoclonal antibodies (MAbs) against caspase-8 (Ab-3), caspase-9 (F-7), and caspase-3 (E-8), as well as rabbit polyclonal antibody against ABCB1 (MDR1), bcl-2 and bak were purchased from Santa-Cruz Biotechnology (Santa Cruz, Calif, USA). PARP (c-20) was purchased from Pharmingen (San Diego, Calif, USA). Peroxidase-conjugated anti-mouse and antirabbit IgG were purchased from Calbiochem (La Jolla, Calif, USA).

2.2. Cell Lines and Cell Culture. The human epidermoid carcinoma cell line $\mathrm{KB}$ and its vincristine-selected derivative KBv200 overexpressing ABCB1 were obtained from Chinese Academy of Medical Sciences, Beijing, and were cultured in RPMI 1640 culture medium with $10 \% \mathrm{FBS}$ at $37^{\circ} \mathrm{C}$ in the presence of $5 \% \mathrm{CO}_{2}[10]$.

2.3. Cytotoxicity Assay. The MTT assay was used to access cytotoxicity as described [11]. Briefly, cells were grown in 96well plates and various concentrations of drugs were added to the wells for 72 hours prior to being assayed. The $\mathrm{IC}_{50}$ values and the degree of resistance were calculated as described in [11]. 
2.4. Apoptotic Cells Detected by Hoechst 33258 Dye. After treatment with bullatacin for 48 hours, the cells were collected, washed with PBS, fixed with $10 \%$ paraformaldehyde for 30 minutes, and incubated in Hoechst $33258(30 \mu \mathrm{g} / \mathrm{mL})$ at room temperature for 30 minutes. Nuclear morphology was examined using fluorescence microscopy. To calculate the percentage of apoptotic cells, all cells from four random microscopic fields at $400 \times$ magnification were counted [12].

2.5. Annexin V-FITC Labeling of Apoptotic Cell Membranes. Annexin V and PI staining was performed using ApopNexinTM FITC Apoptosis Detection Kit (Chemicon, USA). After treated with bullatacin of desired concentrations for 48 hours, both floating and attached cells were collected and resuspended in $200 \mu \mathrm{L} 1 \times$ binding buffer containing Annexin $\mathrm{V}(1: 50)$ and PI ( $40 \mathrm{ng} / \mathrm{sample})$ for 15 minutes at $37^{\circ} \mathrm{C}$ in the dark. Then the numbers of viable, apoptotic, and necrotic cells were quantified by flow cytometer (Becton Dickinson, USA) and analyzed by CellQuest software as described [13]. At least 10,000 cells were analyzed for each sample.

2.6. Preparation of Cytosolic Extracts. The cells were washed with PBS for three times and collected by centrifugation at $500 \times \mathrm{g}$ for 10 minutes. The cell pellet was resuspended in ice-cold cell extract buffer (20 mM HEPES, pH 7.5, 10 $\mathrm{mM} \mathrm{KCl}, 1.5 \mathrm{mM} \mathrm{MgCl}_{2}, 1 \mathrm{mM}$ EDTA, 1 mM EGTA, 1 mM DTT, $250 \mathrm{mM}$ sucrose, protease inhibitor mixture) and incubated for 1 hour at $4^{\circ} \mathrm{C}$. The lysates were then pestled with 30 strokes using a homogenizer. Homogenates were centrifuged at $12,000 \times \mathrm{g}$ for 5 minutes at $4^{\circ} \mathrm{C}$. Supernatants were collected and further centrifuged at $100,000 \times \mathrm{g}$ for 30 minutes at $4^{\circ} \mathrm{C}$ to obtain cytosol [13].

2.7. Immunoblot Analysis. The cells were treated with bullatacin. After treatment, whole-cell lysates were extracted with lysis buffer containing $1 \%$ Triton-100, $50 \mathrm{mM}$ sodium chloride, $50 \mathrm{mM}$ sodium fluoride, $20 \mathrm{mM}$ Tris ( $\mathrm{pH} 7.4$ ), $1 \mathrm{mM}$ EDTA, $1 \mathrm{mM}$ EGTA, $1 \mathrm{mM}$ sodium vanadate, $0.2 \mathrm{mM}$ phenylmethylsulfonyl fluoride, and $0.5 \%$ Nonidet P-40. Western blotting was carried out as described previously [13] and the proteins were detected by the enhanced chemiluminescence detection system (Amersham, Aylesbury, UK).

2.8. Reactive Oxygen Species (ROS) Detection. 2,7-Dichlorodihydrofluorescein diacetate (DCFH-DA) was used as ROS capturing reagent with the method described previously $[13,14]$. DCFH-DA was deacetylated intracellularly by nonspecific esterase, and then furthered oxidized by ROS to the fluorescent compound 2,7-dichlorofluorescein (DCF). Cells were incubated with $10 \mu \mathrm{M}$ DCFH-DA at $37^{\circ} \mathrm{C}$ for 15 minutes. DCF fluorescence was detected by flow cytometry.

2.9. Mitochondrial Transmembrane Potential Measurement. $\Delta \Psi m$ was measured by flow cytometry with the mitochondrial tracking fluorescent compound 3,3'-dihexyloxacarbocynine $\left(\mathrm{DiOC}_{6}\right)$. Uptake and accumulation of $\mathrm{DiOC}_{6}$ in the mitochondrion are driven by $\Delta \Psi m$, therefore the collapse of $\Delta \Psi m$ is reflected by reduction or loss of
DiOC $_{6}$ staining. DiOC $_{6}$ at $100 \mathrm{nmol} / \mathrm{L}$ was added into the cell culture for 15 minutes at $37^{\circ} \mathrm{C}$ and then monitored by flow cytometry with excitation wavelength at $488 \mathrm{~nm}$ and emission wavelength at $530 \mathrm{~nm}[13,15]$.

2.10. Caspase Assays. Activity assays of Caspase- 8 and Caspase- 9 were performed with the ApoAlert Caspase- 8 Colorimetric Assay and the Caspase-9 Fluorescence assay kits (both from Clontech), respectively. Equal numbers of control or bullatacin-treated cells were incubated in cell lysis buffer for 10 minutes, centrifuged, and the supernatants were incubated in reaction buffer that contained IETD-AFC (specific substrate for Caspase-8) or LEHD-AMC (specific substrate for Caspase-9) at $37^{\circ} \mathrm{C}$ for 1 hour. The activity was assayed using a spectrophotometer for Caspase- 8 and a fluorometer for Caspase-9. The specific change of Caspase9 activity was confirmed by the addition of the Caspase9 inhibitor LEHD-CHO before incubation with Caspase- 9 substrate, in parallel experiments [16].

2.11. Statistical Analysis. All experiments were replicated at least three times. Statistical analysis was done by Student's $t$ test analyses. The significance was determined at $P<.05$.

\section{Results}

3.1. KBv200 Cells Are Resistant to Anticancer Drugs. KBv200 cell line is a classical multidrug-resistant human epidermoid carcinoma cell line that upregulates ABCB1 (Figure 1(b)). To determine the resistant property of KBv200 cells, cytotoxicity of anticancer drugs for KBv200 was compared with their parental drug-sensitive KB cells. KB or KBv200 cells were cultured in the presence of full-range concentrations of anticancer drugs such as vincristine, paclitaxel, or doxorubicin for 72 hours, and cell death was determined by MTT assay. The chemotherapeutic agents effectively killed KB cells, whereas KBv200 cells were resistant to the chemotherapeutic agent-induced death (Figures 1(d), 1(e), and 1(f)). KBv200 cells were approximately 62-, 70-, and 75-fold resistant to vincristine, paclitaxel and doxorubicin compared with $\mathrm{KB}$ cells, respectively.

3.2. Inhibition of Cell Growth by Bullatacin in KB and KBv200 Cells. Cytotoxic effects of bullatacin on $\mathrm{KB}$ and $\mathrm{KBv} 200$ cells were measured to examine whether $\mathrm{KB}$ and $\mathrm{KBv} 200$ cells exhibit different sensitivity to bullatacin. It exhibited an inhibitory effect of bullatacin on the proliferation of both KB and KBV200 cells in a concentration-dependent manner after a 72-hour treatment (Figure 1(c)). Similar $\mathrm{IC}_{50}$ of bullatacin was observed in $\mathrm{KB}$ and $\mathrm{KBv} 200$ cells $(2.5 \pm 1.2 \mathrm{nmol} /$ Lversus $2.7 \pm 1.3 \mathrm{nmol} / \mathrm{L}, P>.05)$, suggesting bullatacin is characterized by similar cytotoxicity to the two cell lines.

3.3. Cell Apoptosis Induced by Bullatacin. MDR cells are resistant to apoptosis induced by traditional anticancer drugs. To examine whether ABCB1-positive cells failed to undergo apoptosis induced by bullatacin, apoptosis was 
assessed by Hoechst 33258 staining. Treatment with bullatacin resulted in significant apoptosis in both KB and KBv200 cells with condensed and fragmented nuclei (Figure 2(a)). The apoptotic rate was concentration-dependent after the treatment of 5, 10 and $20 \mathrm{nmol} / \mathrm{L}$ bullatacin for 48 hours (Figure 2(b)).

To further confirm that bullatacin leads to apoptosis, KB and KBv200 cells were stained with Annexin V-FITC and PI, and subsequently analyzed by flow cytometry. As indicated by FCM analysis, the proportion of Annexin V-staining cells was increased with concentration in bullatacin-treated cells (Figure 2(c)); Annexin V-staining KB and KBv200 cells at control group, 5,10 and, $20 \mathrm{nmol} / \mathrm{L}$ bullatacin treating group were $1.8 \%$ versus $1.5 \%, 11.4 \%$ versus $12.9 \%, 23.0 \%$ versus $24.9 \%$ and $48.1 \%$ versus $46.5 \%$, respectively, (Figure $2(\mathrm{~d})$ ).

3.4. ROS Generation and Mitochondrial Transmembrane Potential Loss Induced by Bullatacin. As we know, ABCB1 inhibited the receptor-dependent apoptotic pathway (caspase- 8 as an apical caspase). Did bullatacin induce cell apoptosis via mitochondrial-dependent pathway (caspase9 as an apical caspase)? To examine the mechanism of apoptosis in bullatacin-treated KBv200 cells, we determined ROS by flow cytometry. A concentration-dependent increase of ROS formation was observed after treatment with bullatacin of desired cocentrations for 12 hours (Figure 2(e)).

The $\Delta \Psi m$ loss was measured with $\mathrm{DiOC}_{6}$, which incorporates into mitochondria according to $\Delta \Psi \mathrm{m}$. As shown in Figure 2(f), $\Delta \Psi m$ of KBv200 cells decreased in a concentration-dependent manner after administration of bullatacin for 12 hours.

3.5. Bullatacin Induced Cytochrome C Release and Caspase-9 and -3 Activation in KBv200 Cells. Mitochondrial dysfunction induces cytochrome $\mathrm{c}$ (Cyt $\mathrm{C}$ ) release from the mitochondria to the cytosol. Immunoblotting was performed to test the release of Cyt $\mathrm{C}$ and the subsequent activation of caspase- 9 and -3 . As shown in Figure 3(a), compared with the control, the $15-\mathrm{kDa}$ band representing for cytosolic Cyt $\mathrm{C}$ became clear at $5 \mathrm{nmol} / \mathrm{L}$ and fully developed at $20 \mathrm{nmol} / \mathrm{L}$, implying that Cyt $\mathrm{C}$ was released from the mitochondrial compartment to the cytosol in a concentration-dependent manner after the treatment of bullatacin. The release of Cyt $\mathrm{C}$ from the mitochondrial intermembrane space into the cytosol may be a limiting factor in caspase- 9 activation and represents a central coordinating step in apoptosis. In consistent with what we found, the inactive caspase- 9 and -3 precursors and PARP were cleaved in the presence of bullatacin. However, inactive caspase- 8 precursor was not cleaved in the same condition (Figure 3(a)).

To confirm whether bullatacin specially triggers apical caspase- 9 activation, activities of caspase- 8 and -9 as well as cell apoptosis were assayed after treatment of KBv200 cells with $10 \mathrm{nmol} / \mathrm{L}$ bullatcin for 48 hours in the presence or absence of caspase- 8 inhibitor z-IETD-fmk and caspase9 inhibitor z-LEHD-fmk. As shown in Figures 3(c) and $3(d)$, a significant increase of caspase-9 activity in KBv200 cells treated with bullatacin alone was detected $(P<.01)$. In contrast, activation of caspase- 8 was not observed in the same condition $(P>.05)$. Interestingly, z-LEHDfmk blocked the activation of caspase- 9 and cell apoptosis induced by bullatacin, but z-IETD-fmk did not. These suggested that caspase- 9 and -3 were involved in bullatacininduced apoptosis of KBv200 cells.

Moreover, Bcl-2 and bax are associated with the mitochondrial-dependent apoptosis, whereas Fas and Fas ligand are involved in that of receptor-dependent cell apoptosis. Treatment of KBv200 cells with bullatacin did not cause significant alteration in the expression of bcl-2, bax and Fas (Figure 3(b)).

To study the role of ROS accumulation in the cytotoxicity of bullatacin toward KBv200 cells, we evaluated the combined effect of bullatcin and $\mathrm{N}$-acetyl-l-cysteine (NAC), an antioxidant molecule. The protective effect of NAC against bullatacin-mediated apoptosis was also assessed by measuring Annexin V. Compared with treatment of $10 \mathrm{nmol} / \mathrm{L}$ bullatacin alone for 48 hours, ROS generation and cell apoptosis significantly decreased in KBv200 cells pretreated with $1 \mathrm{mmol} / \mathrm{L}$ NAC for 4 hours (Figures 4 (a) and $4(\mathrm{~b}))$.

\section{Discussion}

Conventional cancer chemotherapy is significantly limited by tumor cells exhibiting MDR, caused by the overexpression of integral membrane transporters, such as ABCB1 and MDR-associated proteins (MRPs/ABCCs), causing decrease of drug accumulation and cellular death. A novel strategy was the development of new anticancer drug with efficacy to MDR cells. Annonaceous acetogenins such as bullatacin and acetogenin 89-2 were potent anticancer agents not only responsive for sensitive cells but also MDR cells in vitro and in vivo [7-9, 17]. ATP depletion is the mode of action of the annonaceous acetogenins, offering a special advantage in the chemotherapeutic treatment of MDR tumors undergoing ATP-dependent mechanisms.

In consistence with our previous findings, bullatacin significantly inhibited cell proliferation and induced apoptosis in $\mathrm{KB}$ and KBv200 cells. Chih et al. [18] reported most of the bullatacin-induced death of human hepatocarcinoma 2.2.15 cell was due to apoptosis. In the present study, we found that bullatacin could induce cell apoptosis not only in drugsensitive KB cells but also in MDR KBv200 cells.

In the mitochondrial-dependent apoptosis pathway, the instability of mitochondria leads to the redistribution of Cyt C into the cytosol, which initiates the formation of the apoptosome and the sequential activation of caspase- 9 and $-3[17,19,20]$. The present study found $\Delta \Psi_{m}$ loss and Cyt $\mathrm{C}$ release into cytosol and the sequential activation of caspase- 9 and -3 in KBv200 cells treated with bullatacin. Furthermore, caspase- 9 inhibitor could particularly block bullatacin-mediated cell apoptosis in KBv200 cells, but not for caspase- 8 inhibitor. These suggest that mitochondrialdependent pathway is involved in bullatacin-trigged MDR cell apoptosis. 


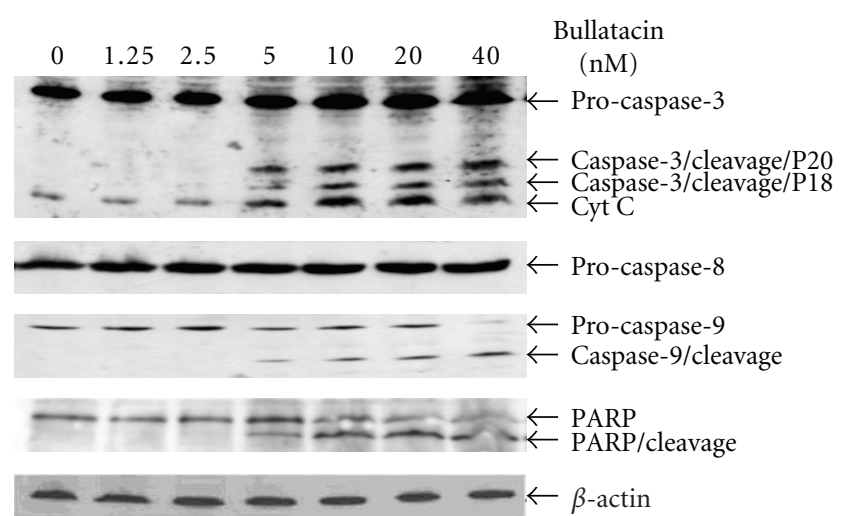

(a)
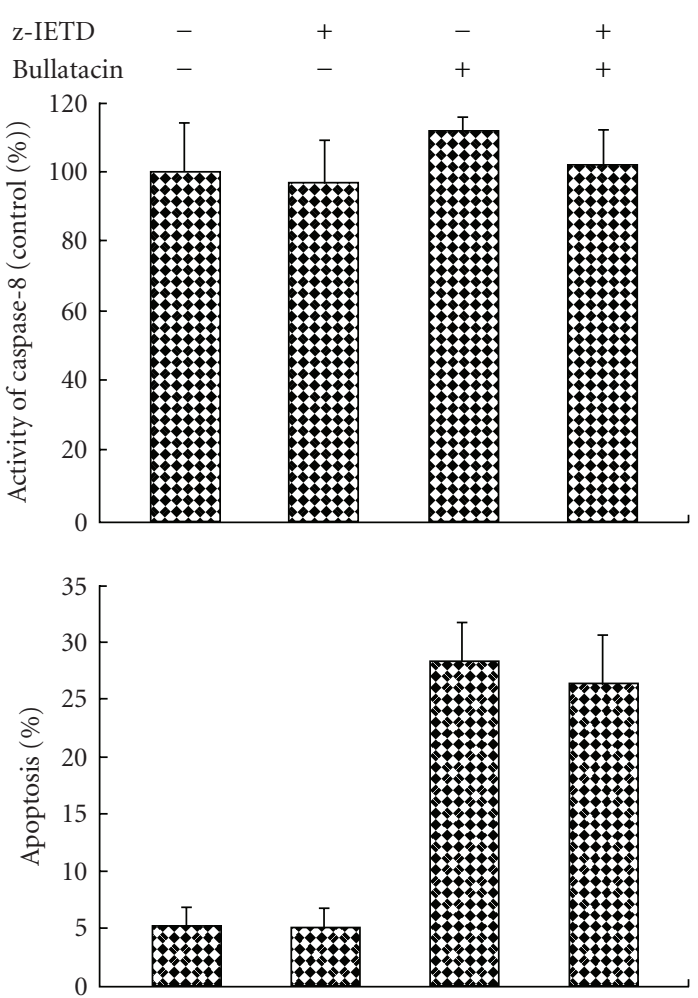

(c)

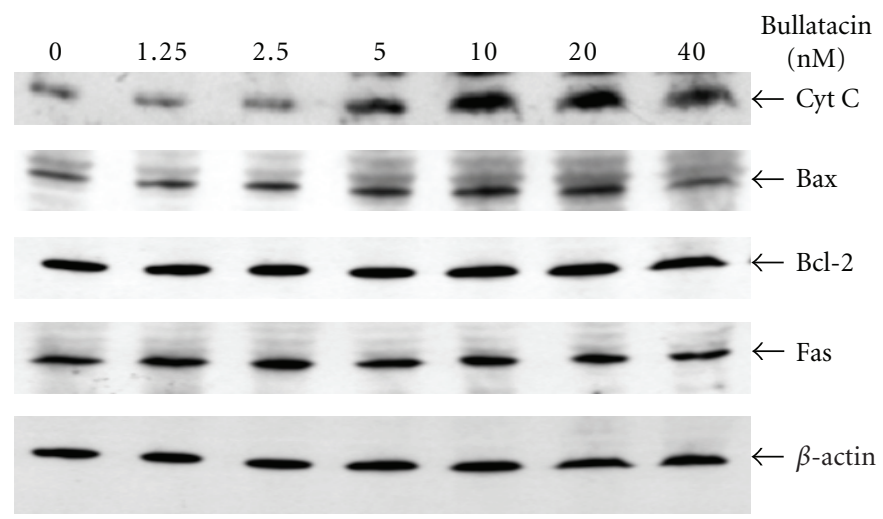

(b)
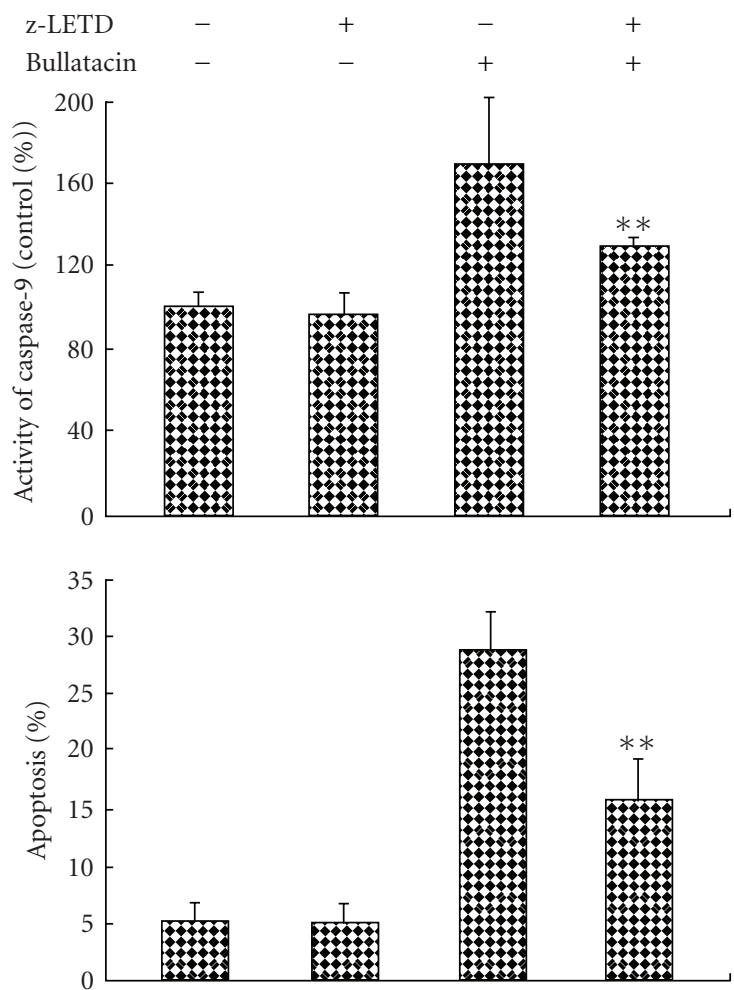

(d)

FIgURE 3: Effect of bullatacin on the release of cytochrome $C$ from mitochondrial, activation of caspases (caspase-9, -8 and -3 ) and cleavage of PARP (a) and on the expression of bax, bcl-2 and Fas (b) in KBv200 cells. After incubation with bullatacin at desired concentrations for 48 $\mathrm{h}$, the cellular proteins were subjected to western blot analysis. The experiments were performed three times and a representative experiment is shown; ( $c$ and d) Effect of bullatacin on capase- 8 and -9 activation and apoptosis induction in KBv200 cells. KBv200 cells were incubated for 12 hours at $37^{\circ} \mathrm{C}$ in the absence or presence of caspase inhibitors, at a concentration of $10 \mu \mathrm{M}$, prior to their exposure to $10 \mathrm{nmol} / \mathrm{L}$ bullatacin. 48 hours later, capase- 8 and -9 activities were examined as the description of Section 2. Data represented means and standard errors of at least a triplicate determination.

Although the downstream events following the release of Cyt C from the mitochondria have been characterized, the early signal-initiating events that cause the instability of mitochondria by bullatacin remain poorly understood. Thus, our effort was to examine the ROS generation that influences the mitochondria function and ultimately elucidate its mechanism of action in KBv200 cells. We found that treating with bullatacin caused the production of ROS, followed by mitochondrial membrane permeabilization and initiation of the apoptotic cascade, including Cyt $\mathrm{C}$ release and caspases activation. Moreover, NAC, an antioxidant agent, decreased ROS generation and protected KBv200 cells from apoptosis induced by bullatacin.

ABCB1, a pump excreting classic anticancer drugs from the inside of MDR cells, inhibits the sequent activation of caspase- 8 and -3 [2], exhibiting an inhibition of apoptosis 

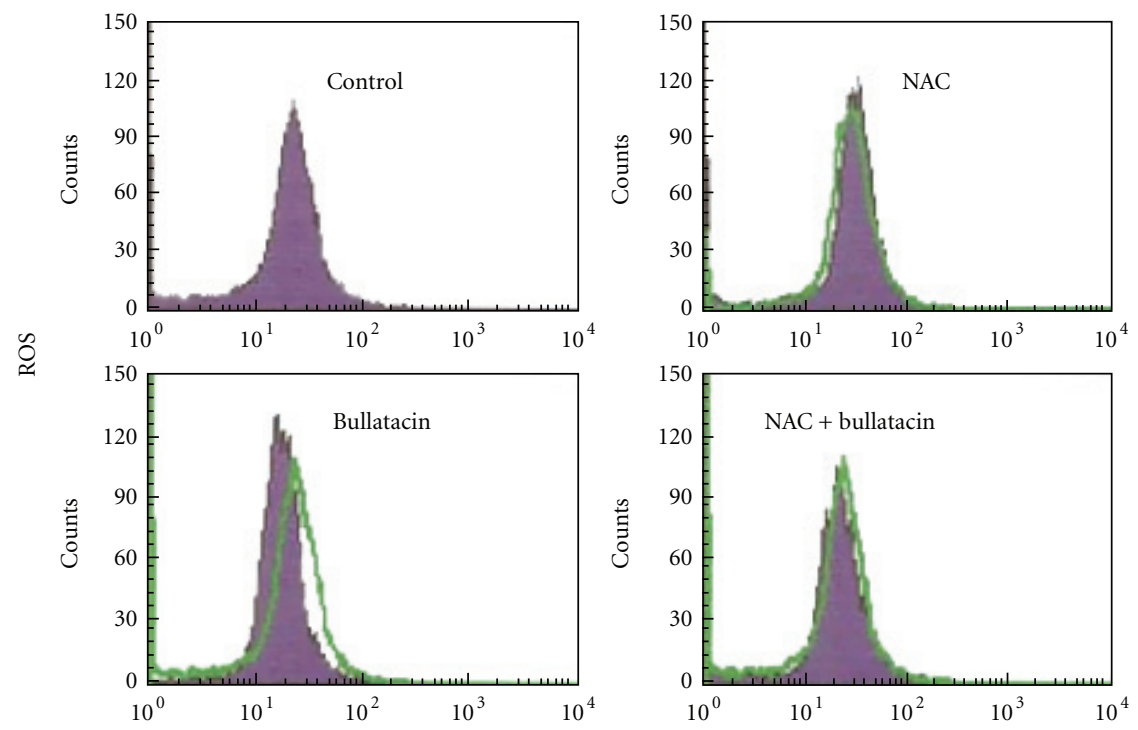

(a)
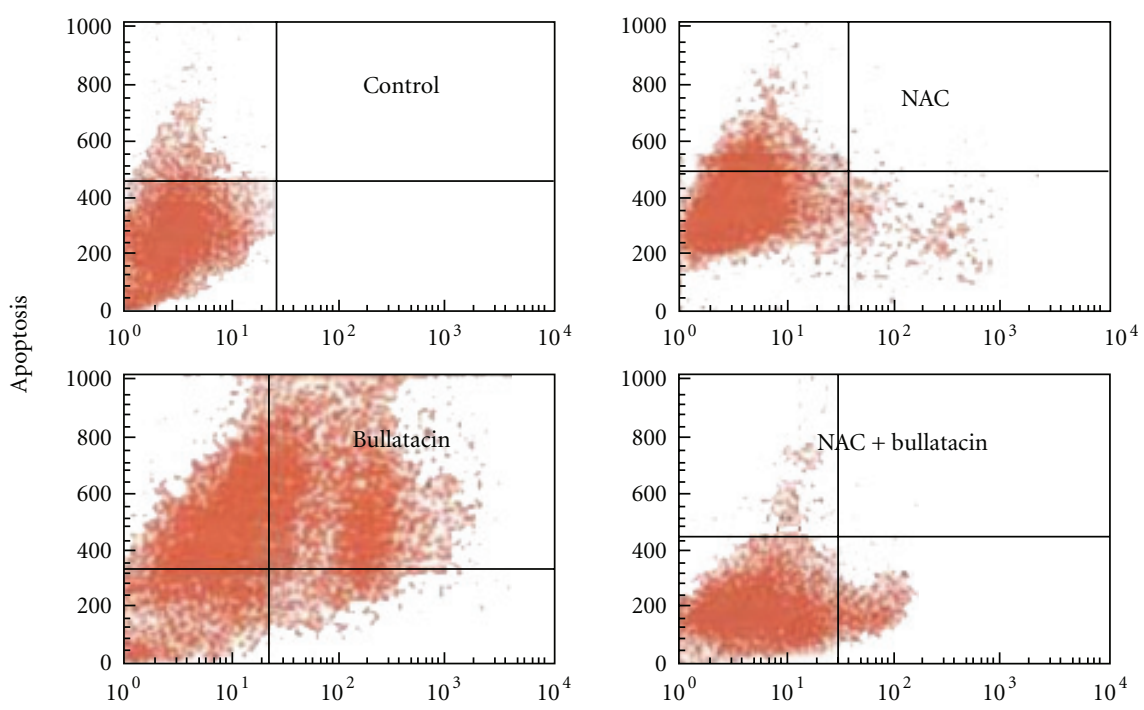

(b)

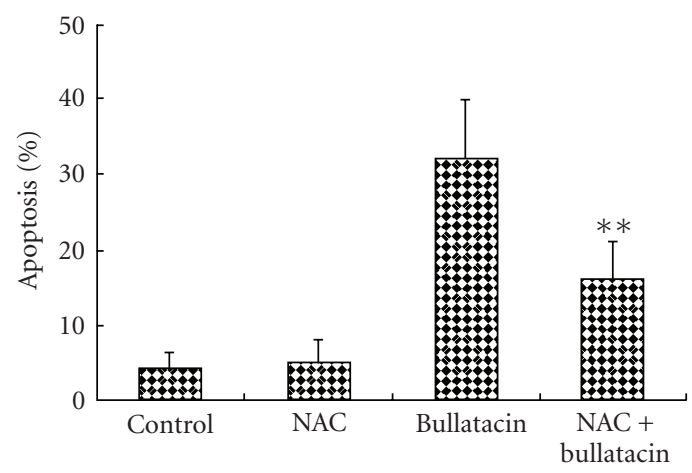

(c)

FIGURE 4: Effect of antioxidant molecules N-acetyl-1-cysteine (NAC) on ROS generation (a) and cell apoptosis (b) induced by bullatacin in KBv200 cells. KBv200 cells pretreated with $1 \mathrm{mmol} / \mathrm{L}$ NAC for 4 hours and then exposed to $10 \mathrm{nmol} / \mathrm{L}$ bullatacin for 48 hours, ROS generation and cell apoptosis were examined. The experiments were performed three times and a representative experiment is shown. 
induced by receptor-dependent pathway. Bullatacin could generate ROS and trigger mitochondria-dependent apoptotic pathway that is not inhibited by ABCB1.

In conclusion, while mitochondrial properties induced by ROS were clearly altered during bullatacin-mediated cell death, our results demonstrate that the mitochondrialdependent pathway is implicated in this cell apoptotic process.

\section{Abbreviations}

MTT: Methylthiazoly1-2,5-diphenylterazolium bromide $\Delta \Psi m$ : Mitochondrial transmembrance potential

ROS: Reactive oxygen species

PARP: Poly(ADP-ribose) polymerase.

\section{Acknowledgments}

We thank Dr. Zhe-Sheng Chen and Tong Shen (Department of Pharmaceutical Sciences, College of Pharmacy and Allied Health Professions, St. John's University) for the editorial assistance. This work was supported by funds from China National Natural Sciences Foundation no. 30672407 (L.-w. $\mathrm{Fu}$ ) and 863 Project Foundation no. 2006AA09Z419 (L.-w. $\mathrm{Fu})$.

\section{References}

[1] K. Takara, T. Sakaeda, and K. Okumura, "An update on overcoming MDR1-mediated multidrug resistance in cancer chemotherapy," Current Pharmaceutical Design, vol. 12, no. 3, pp. 273-286, 2006.

[2] R. W. Johnstone, E. Cretney, and M. J. Smyth, "P-glycoprotein protects leukemia cells against caspase-dependent, but not caspase-independent, cell death," Blood, vol. 93, no. 3, pp. 1075-1085, 1999.

[3] S. H. Kaufmann and D. L. Vaux, "Alterations in the apoptotic machinery and their potential role in anticancer drug resistance," Oncogene, vol. 22, no. 6, pp. 7414-7430, 2003.

[4] G. Galati and P. J. O’Brien, "Potential toxicity of flavonoids and other dietary phenolics: significance for their chemopreventive and anticancer properties," Free Radical Biology and Medicine, vol. 37, no. 3, pp. 287-303, 2004.

[5] J. G. Okun, P. Lummen, and U. Brandt, "Three classes of inhibitors share a common binding domain in mitochondrial complex I (NADH:Ubiquinone oxidoreductase)," The Journal of Biological Chemistry, vol. 274, no. 5, pp. 2625-2630, 1999.

[6] D. J. Morre, R. de Cabo, C. Farley, N. H. Oberlies, and J. L. McLaughlin, "Mode of action of bullatacin, a potent antitumor acetogenin: inhibition of NADH oxidase activity of HeLa and HL-60, but not liver, plasma membranes," Life Sciences, vol. 56, no. 5, pp. 343-348, 1995.

[7] N. H. Oberlies, C.-J. Chang, and J. L. McLaughlin, "Structureactivity relationships of diverse Annonaceous acetogenins against multidrug resistant human mammary adenocarcinoma (MCF-7/Adr) cells," Journal of Medicinal Chemistry, vol. 40, no. 13, pp. 2102-2106, 1997.

[8] N. H. Oberlies, V. L. Croy, M. L. Harrison, and J. L. McLaughlin, "The Annonaceous acetogenin bullatacin is cytotoxic against multidrug-resistant human mammary adenocarcinoma cells," Cancer Letters, vol. 115, no. 1, pp. 73-79, 1997.

[9] L.-W. Fu, L.-R. He, Y.-J. Liang, et al., "Experimental chemotherapy against xenografts derived from multidrug resistant KBv200 cells and parental drug-sensitive $\mathrm{KB}$ cells in nude mice by annonaceous acetogenin 89-2," Acta Pharmaceutica Sinica, vol. 38, no. 8, pp. 565-570, 2003.

[10] L. W. Fu, Y. M. Zhang, Y. J. Liang, X. P. Yang, and Q. C. Pan, "The multidrug resistance of tumour cells was reversed by tetrandrine in vitro and in xenografts derived from human breast adenocarcinoma MCF-7/adr cells," European Journal of Cancer, vol. 38, no. 3, pp. 418-426, 2002.

[11] Z. Shi, Y.-J. Liang, Z.-S. Chen, et al., "Reversal of MDR1/Pglycoprotein-mediated multidrug resistance by vector-based RNA interference in vitro and in vivo," Cancer Biology \& Therapy, vol. 5, no. 1, pp. 39-47, 2006.

[12] K. Hishikawa, B. S. Oemar, F. C. Tanner, T. Nakaki, T. F. Lüscher, and T. Fujii, "Connective tissue growth factor induces apoptosis in human breast cancer cell line MCF-7," The Journal of Biological Chemistry, vol. 274, no. 52, pp. 3746137466, 1999.

[13] X.-H. Wang, D.-Z. Jia, Y.-J. Liang, et al., "Lgf-YL-9 induces apoptosis in human epidermoid carcinoma $\mathrm{KB}$ cells and multidrug resistant KBv200 cells via reactive oxygen speciesindependent mitochondrial pathway," Cancer Letters, vol. 249, no. 2, pp. 256-270, 2007.

[14] Y. Ding, L.-R. He, K.-J. Cao, Y. Lu, L.-Q. Gu, and L.-W. $\mathrm{Fu}$, "Apoptosis of human carcinoma of mouth floor KB cells and multidrug resistant KBv200 cells induced by azide methyl anthraquinone derivative," Acta Pharmaceutica Sinica, vol. 40, no. 1, pp. 22-26, 2005.

[15] S. B. Le, E. L. Holmuhamedov, V. L. Narayanan, E. A. Sausville, and S. H. Kaufmann, "Adaphostin and other anticancer drugs quench the fluorescence of mitochondrial potential probes," Cell Death and Differentiation, vol. 13, no. 1, pp. 151-159, 2006.

[16] T. Fukazawa, B. Walter, and L. B. Owen-Schaub, "Adenoviral Bid overexpression induces caspase-dependent cleavage of truncated Bid and p53-independent apoptosis in human nonsmall cell lung cancers," The Journal of Biological Chemistry, vol. 278, no. 28, pp. 25428-25434, 2003.

[17] J. R. Tormo, N. DePedro, I. Royo, et al., "In vitro antitumor structure-activity relationships of threo/trans/threo/ trans/erythro bis-tetrahydrofuranic acetogenins: correlations with their inhibition of mitochondrial complex I," Oncology Research, vol. 15, no. 3, pp. 129-138, 2005.

[18] H.-W. Chih, H.-F. Chiu, K.-S. Tang, F.-R. Chang, and Y.-C. $\mathrm{Wu}$, "Bullatacin, a potent antitumor annonaceous acetogenin, inhibits proliferation of human hepatocarcinoma cell line 2.2.15 by apoptosis induction," Life Sciences, vol. 69, no. 11, pp. 1321-1331, 2001.

[19] E. Pozo-Guisado, J. M. Merino, S. Mulero-Navarro, et al., "Resveratrol-induced apoptosis in MCF-7 human breast cancer cells involves a caspase-independent mechanism with downregulation of $\mathrm{Bcl}-2$ and NF- $\kappa \mathrm{B}$," International Journal of Cancer, vol. 115, no. 1, pp. 74-84, 2005.

[20] J. Gordon, C.-H. Wu, M. Rastegar, and A. R. Safa, " $\beta 2-$ microglobulin induces caspase-dependent apoptosis in the CCRF-HSB-2 human leukemia cell line independently of the caspase-3, -8 and -9 pathways but through increased reactive oxygen species," International Journal of Cancer, vol. 103, no. 3, pp. 316-327, 2003. 


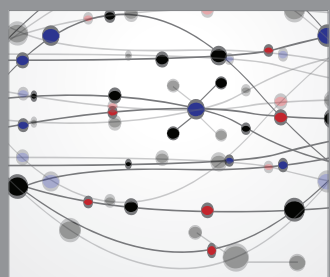

The Scientific World Journal
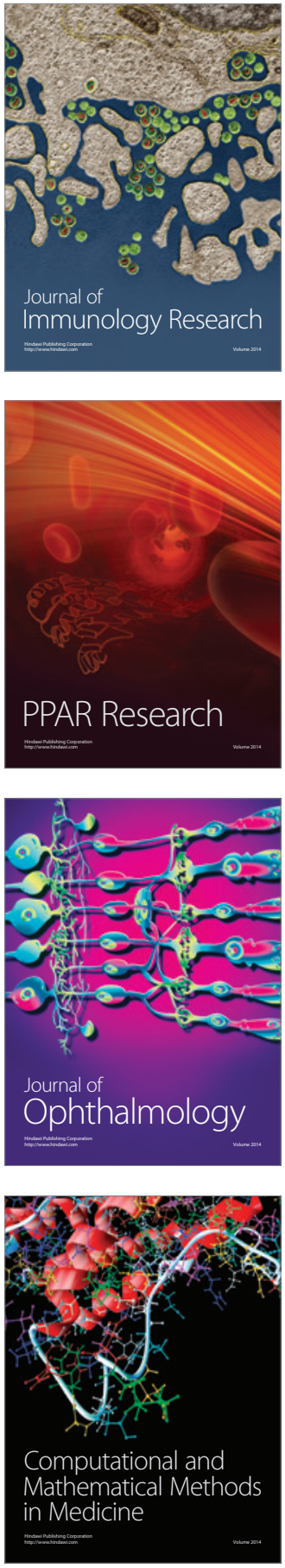

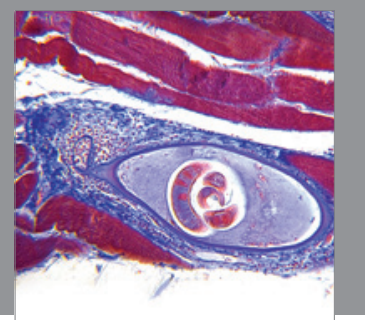

Gastroenterology

Research and Practice
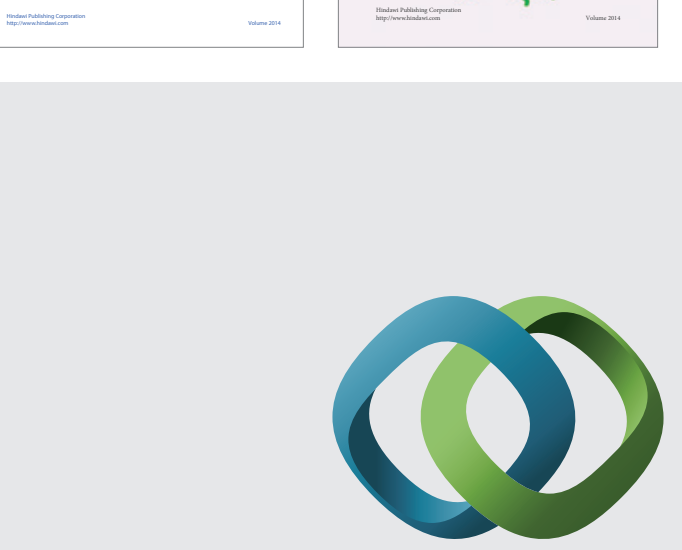

\section{Hindawi}

Submit your manuscripts at

http://www.hindawi.com
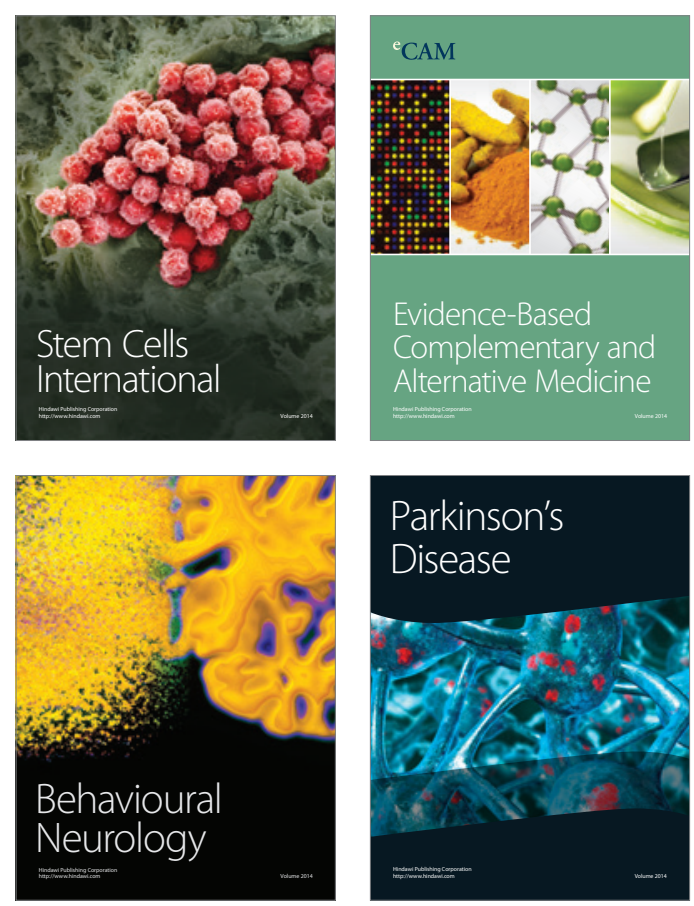

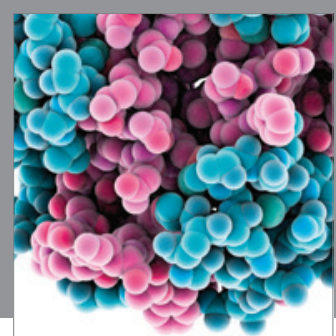

Journal of
Diabetes Research

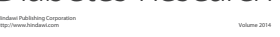

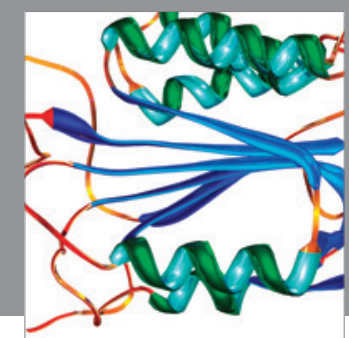

Disease Markers
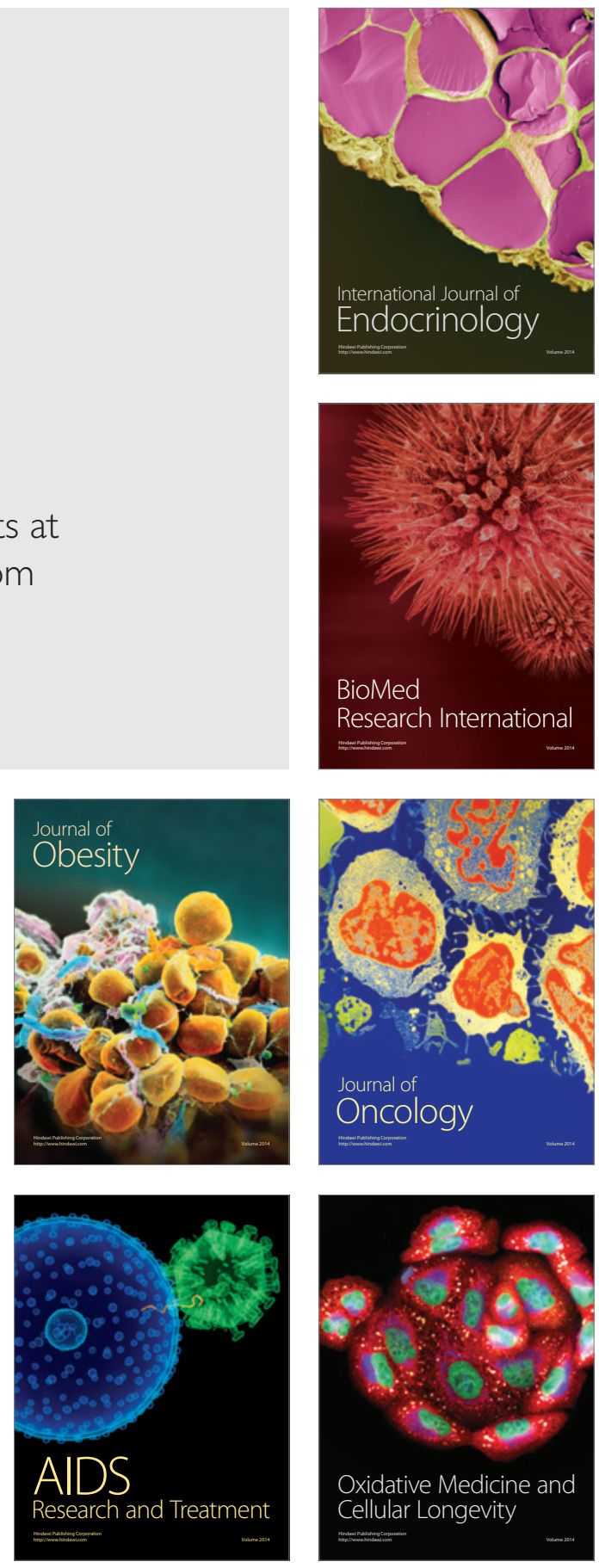